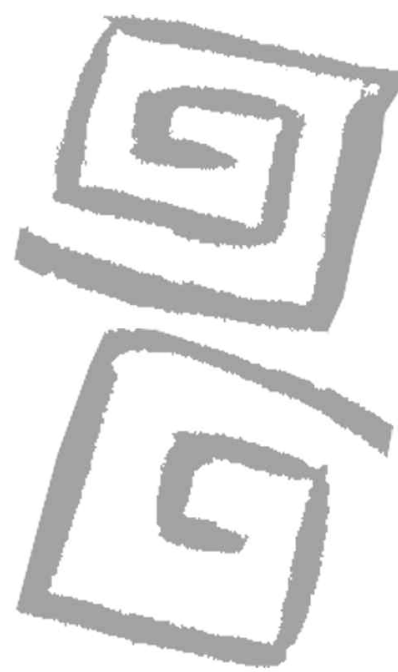

\title{
Actitudes de adolescentes hacia la salud: evaluación de un programa escolar de promoción de la salud en Sevilla, España
}

\author{
Attitudes of adolescents regarding health: evaluation \\ of a school-based health promotion program in \\ Seville, Spain
}

Lima Serrano, Marta'; Neves de Jesus, Saul2;

Lima Rodríguez, Joaquín Salvador ${ }^{3}$

\footnotetext{
${ }^{1}$ Licenciada en Antropología Social y Cultural. Diploma de Estudios Avanzados en Enfermería. Profesora Ayudante de Enfermería, Universidad de Sevilla, España. mlima@us.es

2Licenciado en Psicología. Doctor en Psicología de la Educación. Profesor Catedrático de Psicología, Universidad de Algarve, Portugal. snjesus@ualg.pt

3 Licenciado en Antropología Social y Cultural. Doctor en Enfermería. Profesor Titular de Enfermería, Universidad de Sevilla, España. joaquinlima@us.es
}

RESUMEN Se examinaron diferencias en las actitudes hacia la salud en el alumnado de cuarto año de educación secundaria obligatoria de Sevilla, el cual fue dividido en dos grupos en función de su participación en el programa "Forma Joven" de promoción de la salud. Se realizó un análisis descriptivo y bivariante. No se observaron diferencias significativas en las características sociodemográficas de los dos grupos, considerándose homogéneos. Las actitudes también fueron similares en ambos; únicamente se encontraron diferencias estadísticas en el grado de peligrosidad asignado a dos de las trece situaciones viales expuestas: "no usar el cinturón de seguridad" e "ir en un vehículo cuando el conductor ha bebido", siendo menor el asignado por los participantes en el programa. Esto coincide con lo verificado en trabajos previos e invita a reflexionar sobre la falta de efectividad de este tipo de programas. En el caso del programa "Forma Joven" podría atribuirse a una deficiente sistematización en su implementación. Sería recomendable proponer intervenciones para aumentar su efectividad.

PALABRAS CLAVE Promoción de la salud; Adolescentes; Evaluación de Programas; Conducta de Riesgo; Actitud Frente a la Salud.

ABSTRACT The study examined differences in attitudes regarding health within students in their fourth year of mandatory secondary school in Seville, Spain. The students were divided into two groups according to their participation in the "Forma Joven" health promotion program. A descriptive and bivariate analysis was conducted. As there were no significant differences in the socio-demographic characteristics of the two groups, the groups were considered homogeneous. Attitudes were also similar in both groups. Statistically significant differences were only found in the level of danger assigned to two of the thirteen transit situations explored: "not using a seatbelt" and "entering a vehicle when the driver has been drinking," with the lower level of risk corresponding to the group of students participating in the program. These findings are consistent with the results of previous studies and invites reflection upon the effectiveness of such programs. In the case of the "Forma Joven" program, the ineffectiveness could be attributable to a lack of systematization in the program's implementation. In the future, interventions to increase the effectiveness of the program should be proposed.

KEY WORDS Health Promotion; Adolescents; Program Evaluation; Risk Behavior; Attitude to Health. 


\section{INTRODUCCIÓN}

Los accidentes de tráfico fueron la primera causa de muerte en Andalucía (España) para el grupo de 15 a 24 años, en 2001 (1). En cuanto al número de víctimas involucradas, por cada víctima mortal había alrededor de 6 heridos graves y 29 leves. La mayor incidencia de accidentes está asociada al consumo de alcohol y otras drogas (2). El consumo de sustancias adictivas tiene también un alto costo en enfermedades, en términos sociales y económicos (3-5). En Andalucía, en una investigación realizada en 2006 con adolescentes de 11 a 17 años, se halló que la edad media de inicio al consumo de tabaco y alcohol era de 13,5 años, y que el 36,2\% había consumido tabaco y el $57,6 \%$ había consumido alcohol. El consumo de sustancias no legales fue inferior: el $8,5 \%$ había consumido cannabis en alguna ocasión y alrededor del 1\% había consumido otras drogas (6).

El $20 \%$ de los adolescentes andaluces de entre 11 y 17 años que mantuvieron relaciones sexuales no utilizaron preservativos y un $5,2 \%$ no usaron ningún método anticonceptivo. Un 2,5\% de chicas había tenido al menos un embarazo, siendo la edad de mayor riesgo los 1516 años (7). Con relación a la interrupción voluntaria del embarazo, se observa una tendencia evolutiva creciente (8). El sida fue la tercera causa de muerte en el año 2001 para jóvenes de 25 a 34 años, pero el contagio había ocurrido varios años antes de su muerte (1).

La obesidad es considerada como la epidemia del siglo XXI (9). En el año 2003, la prevalencia de sobrepeso entre 16 y 24 años fue del $15,4 \%$ y la de obesidad del 3,1\% (10).

Todo estas situaciones se vinculan con lo relacional y lo comportamental, y su reducción requiere de estrategias de promoción de la salud y prevención del riesgo que involucran a distintos sectores (1).

La mayoría de los programas de promoción de la salud en la escuela tienen un enfoque centrado en el problema (consumo de drogas, prevención del embarazo e infecciones de transmisión sexual, etc.), que con frecuencia resultan de iniciativas descoordinadas, fragmentadas y poco eficaces. Se deberían implementar, desde un enfoque más global, programas que se centren en el desarrollo positivo de niños y adolescentes, con el objeto de generar competencias cognitivas, personales, emocionales, morales y sociales que permitan el "empoderamiento" de estos grupos poblacionales y su contribución positiva a la comunidad (11-13).

En el curso académico 2001-2002 se inició el programa "Forma Joven" para promover la salud de los jóvenes y prevenir conductas de riesgo. Desde un enfoque intersectorial, su principal objetivo es establecer puntos de atención en los espacios frecuentados por adolescentes, mayoritariamente institutos de educación secundaria, siendo la población diana aquellos matriculados en los cursos de primero a cuarto año de educación secundaria obligatoria (3). En el curso académico 2009-2010 existían en Andalucía 778 puntos del programa "Forma Joven", 41 en Sevilla capital y 113 en la provincia $(14,15)$, en los que se desarrollaron un conjunto de actividades de promoción y educación para la salud, con un equipo de profesionales de salud y de educación conformado habitualmente por un/a enfermero/a del centro de atención primaria y un/a orientador/a del instituto de educación secundaria, al que se sumaron otros agentes sociales y jóvenes que actuaron como mediadores.

Las principales áreas de intervención son: estilos de vida (alimentación equilibrada y actividad física, consumos adictivos, seguridad vial), sexualidad y relaciones afectivas, salud mental, convivencia entre iguales y prevención de la violencia (1). El equipo propone actividades fundamentalmente extracurriculares, siendo la principal la asesoría individual y/o grupal a los adolescentes quienes, previa cita y de forma anónima, plantean sus dudas y reciben información y asesoramiento. Si se detecta una problemática especial, se realiza un seguimiento y pueden ser derivados a otras instituciones. Las asesorías se desarrollan con una frecuencia semanal, bisemanal o mensual, dependiendo de cada instituto y del volumen de demandas existentes. El resto de las actividades son fundamentalmente talleres con grupos definidos (por ejemplo, un grupo de cuarto año de educación secundaria obligatoria, que abordan temáticas relacionadas con las principales áreas de intervención, en función de las necesidades detectadas. Se utiliza una metodología 
expositiva y participativa y, en ocasiones, el tema es previamente abordado por los tutores.

Además, se organizan jornadas de salud, o concursos, entre otras actividades, en las que, con frecuencia, participan agentes sociales, tales como delegaciones de juventud y/o de la mujer de los ayuntamientos, organizaciones no gubernamentales (ONG) de la zona, centros de drogodependencias, policía, guardia civil, etc. Estas actividades no suelen ser evaluadas y si lo son, la evaluación se realiza en forma de valoración o encuesta de satisfacción a los tutores, a los que se les pregunta sobre la experiencia, propuestas de mejora, etc. A pesar de que existe un registro de recolección de datos que permitiría analizar el seguimiento y la evaluación del proceso, todavía no existen evaluaciones de las repercusiones del programa "Forma Joven" sobre la salud de sus destinatarios (1). La evaluación del efecto o impacto de un programa permite examinar las mejoras alcanzadas en la población como resultado de la intervención (16). En el caso del programa "Forma Joven" podría traducirse en conseguir que los adolescentes desarrollen actitudes y conductas promotoras de salud frente a los principales riesgos.

Este trabajo se realizó con el objetivo de conocer las actitudes relacionadas con la alimentación, la sexualidad, las sustancias adictivas y la seguridad vial de adolescentes escolarizados en cuarto año de educación secundaria obligatoria en el área urbana de Sevilla, España, y determinar si existen diferencias en las actitudes entre los escolares que formaron parte del grupo de intervención -participantes en el programa "Forma Joven"- y aquellos que formaron parte del grupo control, no participantes en el programa.

\section{MATERIAL Y MÉTODOS}

Se utilizó un diseño evaluativo para analizar los efectos del programa "Forma Joven", cuasi experimental y post intervención, con un grupo de intervención que había recibido el programa y un grupo de control que no lo había recibido (17). Cuando el programa objeto de evaluación ya se encuentra instaurado, puede evaluarse su impacto comparando el alumnado participante en el mismo, con un grupo control, tratando de controlar estadísticamente algunas variables que pudieran influir en la adopción de conductas de riesgo (18). Se utilizaron medidas para realizar un "control interno" o de emparejamiento de grupos, conformando los grupos lo más similares posibles entre sí (19).

Por la forma en que el programa se desarrolla, se asumió que el alumnado de cuarto año de educación secundaria obligatoria habría recibido en mayor grado sus efectos. La población de estudio fue la de adolescentes de entre 15 y 17 años escolarizados en institutos de educación secundaria de Sevilla capital. La muestra se tomó a partir de los 50 institutos de titularidad pública existentes (20), de los cuales 38 participaban en el programa (15). Se seleccionaron ocho institutos: cuatro formarían el grupo de intervención y cuatro el grupo control. Se establecieron los siguientes criterios de inclusión:

- Heterogeneidad: la presencia del programa "Forma Joven" en los institutos incluidos en el grupo de intervención, o la ausencia de dicho programa en los institutos incluidos en el grupo control.

- Homogeneidad: que los institutos fueran de gestión pública, que fueran alumnos de cuarto año de educación secundaria obligatoria, y que los centros de ambos grupos pertenecieran a la misma demarcación geográfica, teniendo en cuenta la distribución por barrios.

En el caso de los institutos incluidos en el grupo de intervención, se estableció como condición que el programa haya sido implementado en al menos dos cursos académicos previos a la realización del estudio y que existieran registros de seguimiento del proceso. Se excluyeron los institutos localizados fuera del área urbana y aquellos cuyos responsables no desearon participar. La muestra se obtuvo aleatoriamente por conglomerados, escogiendo un grupo de cuarto año de cada uno de los centros. La muestra inicial fue de 166 adolescentes, de los que se recogió la información. Tras la exclusión de aquellos que no se adecuaban por falta de respuestas (menor al 75\%) o incoherencia de las mismas, la distribución final fue de 73 en el grupo de intervención y 73 en el grupo control $(\mathrm{N}=146)$. 
La variable independiente fue la presencia/ausencia del programa en los institutos. Como variables dependientes se exploraron actitudes hacia la alimentación, la sexualidad, las sustancias adictivas y la seguridad vial.

En la bibliografía se identificaron factores de riesgo y de protección para la adopción de conductas de riesgo, y se decidió controlar las siguientes variables: edad, sexo/género, nivel de estudios de las madres y de los padres, nivel socioeconómico familiar percibido y dinámica familiar (21-25).

Al no encontrar ningún instrumento validado acorde con el objetivo del estudio, se utilizó un cuestionario de elaboración propia. Para su construcción se tuvo en cuenta el marco teórico de referencia, así como cuestionarios previamente validados (26-28). Fue pilotado sobre 22 adolescentes de características similares a los participantes para analizar la adecuación, la comprensión, la extensión y la existencia o no de cansancio. El instrumento final contaba con 85 ítems, divididos en cinco subescalas. Las tres primeras valoraban de forma dicotómica (acuerdo/desacuerdo) actitudes respecto a la alimentación (del tipo "las comidas rápidas permiten aprovechar mejor el tiempo"), a la sexualidad (como "prefiero mantener relaciones sexuales con penetración, aunque pueda contagiar$\mathrm{me}^{\prime \prime}$ ) y a las sustancias adictivas ("las personas que beben son más interesantes").

Desde la consideración de que la percepción del riesgo, entendida como el grado en que se atribuye a una conducta un supuesto peligro para la salud, interviene en la adopción de conductas $(29,30)$, se construyeron dos escalas tipo Likert de cuatro puntos, para estudiar el grado de peligrosidad asignado al consumo de sustancias adictivas, y el grado de peligrosidad asignado a determinadas situaciones de seguridad vial (por ejemplo "ir en un vehículo cuando el que conduce ha consumido alcohol").

Para controlar la homogeneidad de los grupos, se incluyeron cinco preguntas sobre características sociodemográficas y el test de Apgar familiar para el estudio de la funcionalidad familiar (31).

El estudio fue aprobado por el Comité de Experimentación de la Universidad de Sevilla. Se garantizó el anonimato, la confidencialidad de la información y la protección de los datos solicitando el consentimiento informado a los participantes, que se consideraron competentes para proporcionarlo, dado el tipo de estudio y la edad de los mismos.

La recolección de la información fue realizada entre mayo y junio de 2009, siguiendo las indicaciones del estudio Health Behavior in School-Age Children (Estudio sobre las conductas saludables de los jóvenes escolarizados), las que señalan que: los propios estudiantes deben ser quienes respondan al cuestionario, el anonimato debe ser garantizado, y la administración de los cuestionarios debe realizarse en el contexto escolar por personal entrenado para ello. Por lo tanto, el cuestionario fue entregado a los alumnos por una investigadora que les informaba acerca de las características de la investigación y de la forma de cumplimentar el cuestionario. Los alumnos posteriormente realizaban las encuestas de forma autoadministrada.

Se realizó un análisis descriptivo de las variables de estudio y un análisis bivariante para comparar los grupos. Se utilizo el test $\mathrm{Chi}^{2}$ de Pearson $\left(\chi^{2}\right)$ para evaluar la significación estadística de las diferencias de proporciones $(p<0,05)$, para comprobar la homogeneidad de los grupos con relación a las variables controladas y para identificar las diferencias en función de la variable independiente (presencia/ausencia del programa en el instituto). Se midió el tamaño del efecto mediante el coeficiente de contingencia $(r \varphi)$ : bajo, $r \varphi=0,1$; medio, $\mathrm{r} \varphi=0,3$; alto, $\mathrm{r} \varphi=0,5$. Los datos perdidos no fueron imputados en el análisis. Se utilizó el programa estadístico SPSS 17.0.

\section{RESULTADOS}

Al realizar las pruebas de homogeneidad de los grupos, no se hallaron diferencias estadísticamente significativas en la distribución de las variables sociodemográficas ni en la funcionalidad familiar de los participantes, por lo que se consideraron homogéneos (Cuadro 1). Para comparar las variables "estudios del padre" y "estudios de la madre" se eliminó la categoría "ninguno" (su frecuencia esperada fue menor al 5\%), y en la variable "percepción de la economía familiar", se agrupó la categoría "en casa tenemos problemas económicos" con "nos arreglamos pero apenas Ilegamos a fin de mes". 
Cuadro 1. Características sociodemográficas de los adolescentes participantes en el estudio, en función de su pertenencia al grupo de intervención o al grupo control. Porcentajes y frecuencias absolutas. Sevilla, 2009.

\begin{tabular}{|c|c|c|c|c|c|c|}
\hline \multirow[t]{2}{*}{ Variables } & \multicolumn{2}{|c|}{$\begin{array}{c}\text { Grupo de } \\
\text { intervención } \\
(n=73)\end{array}$} & \multicolumn{2}{|c|}{$\begin{array}{l}\text { Grupo } \\
\text { control } \\
(n=73)\end{array}$} & \multirow[t]{2}{*}{$\chi^{2}(\mathrm{df}, \mathrm{N})^{\mathrm{a}}$} & \multirow[t]{2}{*}{ Valor de $p$} \\
\hline & $\mathrm{n}$ & $\%$ & $n$ & $\%$ & & \\
\hline \multicolumn{7}{|l|}{ AÑO DE NACIMIENTO } \\
\hline 1991 & 4 & 5,5 & 8 & 11,0 & \multirow{4}{*}{$2,26(2,145)$} & \multirow{4}{*}{0,322} \\
\hline 1992 & 17 & 23,3 & 21 & 28,8 & & \\
\hline 1993 & 51 & 69,9 & 44 & 60,3 & & \\
\hline Sin información & 1 & 1,3 & 0 & 0,0 & & \\
\hline \multicolumn{7}{|l|}{ SEXO } \\
\hline Femenino & 38 & 52,1 & 36 & 49,3 & \multirow[b]{2}{*}{$0,11(1,146)$} & \multirow[b]{2}{*}{0,741} \\
\hline Masculino & 35 & 47,9 & 37 & 50,7 & & \\
\hline \multicolumn{7}{|l|}{ ESTUDIOS DE LA MADRE } \\
\hline Ninguno & 3 & 4,1 & 2 & 2,7 & \multirow{5}{*}{$0,50(2,122)$} & \multirow{5}{*}{0,778} \\
\hline Primarios & 28 & 38,4 & 30 & 41,1 & & \\
\hline Secundarios & 22 & 31,5 & 19 & 26,1 & & \\
\hline Universitarios & 12 & 16,4 & 10 & 13,7 & & \\
\hline Sin información & 7 & 9,6 & 12 & 16,4 & & \\
\hline \multicolumn{7}{|l|}{ ESTUDIOS DEL PADRE } \\
\hline Ninguno & 2 & 2,7 & 3 & 4,1 & \multirow{5}{*}{$1,21(2,123)$} & \multirow{5}{*}{0,547} \\
\hline Primarios & 25 & 34,3 & 28 & 38,4 & & \\
\hline Secundarios & 19 & 26,0 & 18 & 24,7 & & \\
\hline Universitarios & 14 & 19,2 & 9 & 12,3 & & \\
\hline Sin información & 13 & 17,8 & 15 & 20,5 & & \\
\hline \multicolumn{7}{|c|}{ PERCEPCIÓN DE LA ECONOMÍA FAMILIAR } \\
\hline Estamos bastante bien & 41 & 56,1 & 43 & 58,9 & \multirow{3}{*}{$0,11(1,146)$} & \multirow{3}{*}{0,738} \\
\hline $\begin{array}{l}\text { Nos arreglamos pero apenas } \\
\text { llegamos a fin de mes }\end{array}$ & 27 & 37,0 & 29 & 39,7 & & \\
\hline Tenemos problemas económicos & 5 & 6,9 & 1 & 1,4 & & \\
\hline \multicolumn{7}{|l|}{ FUNCIONALIDAD FAMILIAR } \\
\hline Funcionales & 57 & 78,1 & 55 & 75,3 & \multirow{3}{*}{$0,59(1,145)$} & \multirow{3}{*}{0,808} \\
\hline Disfuncionales & 16 & 21,9 & 17 & 23,3 & & \\
\hline Sin información & 0 & 0,0 & 1 & 1,4 & & \\
\hline
\end{tabular}

Fuente: Elaboración propia a partir de los datos recogidos en el estudio.

$\mathrm{a}_{\mathrm{El}} \chi^{2}$ se calcula sobre los datos con información.

No se encontraron diferencias estadísticamente significativas entre el grupo de intervención y el grupo control en ninguna de las actitudes analizadas respecto a la alimentación, la sexualidad y las sustancias adictivas.

En ambos grupos, la mayor parte de las actitudes analizadas fueron positivas. En cuanto a la alimentación, se destacó como negativo que más de la mitad de los estudiantes opinaba que "las comidas rápidas permiten aprovechar mejor el tiempo"; también es importante el porcentaje de aquellos a los que les parecía bien tomar dulces o bollería a diario, y los que afirmaban que "cuando uno/a hace una dieta es normal que se salte alguna comida" (Cuadro 2).

Respecto a la sexualidad no se encontraron diferencias estadísticamente significativas, aunque sí una tendencia en algunos de los ítems. 
Cuadro 2. Grado de acuerdo con actitudes relacionadas con la alimentación de los participantes, en función de su pertenencia al grupo intervención o al grupo control. Porcentajes y frecuencias absolutas. Sevilla, 2009.

\begin{tabular}{|c|c|c|c|c|c|c|}
\hline \multirow[t]{2}{*}{ Actitudes } & \multicolumn{2}{|c|}{$\begin{array}{c}\text { Grupo de } \\
\text { intervención } \\
(n=73)\end{array}$} & \multicolumn{2}{|c|}{$\begin{array}{l}\text { Grupo } \\
\text { control } \\
(\mathrm{n}=73)\end{array}$} & \multirow[t]{2}{*}{$\chi^{2}(\mathrm{df}, \mathrm{N})^{\mathrm{a}}$} & \multirow[t]{2}{*}{ Valor de $p$} \\
\hline & $\mathrm{n}$ & $\%$ & $\mathrm{n}$ & $\%$ & & \\
\hline $\begin{array}{l}\text { Los alimentos preparados en casa son } \\
\text { mejores que los preparados en } \\
\text { hamburgueserías y pizzerías }\end{array}$ & 66 & 90,4 & 66 & 90,4 & $0,07(1,145)$ & 0,791 \\
\hline $\begin{array}{l}\text { Las comidas rápidas permiten } \\
\text { aprovechar mejor el tiempo }\end{array}$ & 35 & 47,9 & 43 & 58,9 & $1,55(1,145)$ & 0,214 \\
\hline $\begin{array}{l}\text { Para llevar una alimentación } \\
\text { saludable he de tomar alimentos } \\
\text { variados (frutas, verduras, carne, } \\
\text { pescado, huevos, lácteos) }\end{array}$ & 70 & 95,9 & 72 & 98,6 & * & 0,310 \\
\hline $\begin{array}{l}\text { Es importante realizar, al menos, tres } \\
\text { comidas diarias }\end{array}$ & 67 & 91,8 & 68 & 93,2 & $0,10(1,146)$ & 0,754 \\
\hline $\begin{array}{l}\text { Es fundamental desayunar antes de } \\
\text { acudir a clase }\end{array}$ & 62 & 84,9 & 67 & 91,8 & $1,66(1,146)$ & 0,197 \\
\hline $\begin{array}{l}\text { Me parece bien tomar dulces o } \\
\text { bollería a diario }\end{array}$ & 16 & 21,9 & 12 & 16,7 & $0,08(1,144)$ & 0,400 \\
\hline Tomar chucherías es adecuado & 10 & 13,7 & 7 & 9,6 & $0,55(1,145)$ & 0,457 \\
\hline $\begin{array}{l}\text { La mejor forma de adelgazar es } \\
\text { realizar una dieta estricta }\end{array}$ & 24 & 32,9 & 20 & 27,4 & $0,45(1,143)$ & 0,503 \\
\hline $\begin{array}{l}\text { Cuanto más delgada está una } \\
\text { persona, más sana }\end{array}$ & 10 & 13,7 & 5 & 6,8 & $1,86(1,146)$ & 0,173 \\
\hline $\begin{array}{l}\text { Cuando uno/a hace dieta es normal } \\
\text { que se salte alguna comida }\end{array}$ & 27 & 37,0 & 22 & 30,1 & $0,58(1,144)$ & 0,447 \\
\hline
\end{tabular}

Fuente: Elaboración propia a partir de los datos recogidos en el estudio.

$\mathrm{a}_{\mathrm{El}} \chi^{2}$ se calcula sobre los datos con información.

*El $50 \%$ de las casillas tiene una frecuencia esperada menor a 5. Se usó el test exacto de Fisher.

Sorprendentemente, es mayor el porcentaje de acuerdo de los estudiantes pertenecientes al grupo de intervención en las siguientes afirmaciones: "a mi chico/a no le gusta que use preservativo", "prefiero mantener relaciones sexuales sin condón, aunque me arriesgue a contraer una enfermedad", y "prefiero mantener relaciones sexuales con penetración, aunque pueda contagiarme".

Con respecto a las actitudes relacionadas con la sexualidad, en ambos grupos, se destacó como negativo que un importante número de estudiantes opinaba que "el uso del preservativo es incómodo porque se siente menos", o que "el hombre es el responsable de la protección" (Cuadro 3).

Con relación al consumo de sustancias, respecto al tabaco, se destacó como negativo el importante número de adolescentes que consideraba que "fumar está de moda" y como positivo que a la mayoría le molestaba su consumo. En el caso del alcohol fue mayor el número de estudiantes con actitudes favorables a su consumo, y por lo tanto negativas, entre las que se destacan las siguientes: "beber alcohol hace sentirse bien", "ayuda a hacer amigos", y "está de moda". Solo a un pequeño porcentaje le molestaba que los demás beban (Cuadro 4).

Respecto al consumo de tranquilizantes, fueron menores los porcentajes de actitudes favorables a su consumo. Se destacó como negativo el porcentaje de estudiantes que opinaba que "hacen sentirse bien" y solo al 28,8\% le molestaba su consumo. Con relación al consumo de hachís se destacaron como negativos los 
Cuadro 3. Grado de acuerdo con actitudes respecto a la sexualidad de los participantes, en función de su pertenencia al grupo intervención o al grupo control. Porcentajes y frecuencias absolutas. Sevilla, 2009.

\begin{tabular}{|c|c|c|c|c|c|c|}
\hline \multirow[t]{2}{*}{ Actitudes } & \multicolumn{2}{|c|}{$\begin{array}{c}\text { Grupo de } \\
\text { intervención } \\
(n=73)\end{array}$} & \multicolumn{2}{|c|}{$\begin{array}{l}\text { Grupo } \\
\text { control } \\
(n=73)\end{array}$} & \multirow[t]{2}{*}{$\chi^{2}(\mathrm{df}, \mathrm{N})^{\mathrm{a}}$} & \multirow[t]{2}{*}{ Valor de $p$} \\
\hline & $n$ & $\%$ & $n$ & $\%$ & & \\
\hline $\begin{array}{l}\text { La primera vez es muy difícil quedarse } \\
\text { embarazada }\end{array}$ & 10 & 13,7 & 6 & 8,2 & $1,12(1,146)$ & 0,289 \\
\hline $\begin{array}{l}\text { Si no se llega al orgasmo no se puede } \\
\text { quedar embarazada }\end{array}$ & 7 & 9,6 & 6 & 8,2 & $0,84(1,146)$ & 0,771 \\
\hline $\begin{array}{l}\text { La marcha atrás es un método eficaz } \\
\text { para evitar el embarazo }\end{array}$ & 14 & 19,2 & 9 & 12,3 & $1,29(1,146)$ & 0,256 \\
\hline $\begin{array}{l}\text { El uso de condón es bueno para mi } \\
\text { salud }\end{array}$ & 69 & 94,5 & 69 & 94,5 & --- & --- \\
\hline $\begin{array}{l}\text { El uso del preservativo es incómodo } \\
\text { porque se siente menos }\end{array}$ & 24 & 32,9 & 24 & 32,9 & --- & --- \\
\hline $\begin{array}{l}\text { La mujer es la que debe solicitar el uso } \\
\text { del condón }\end{array}$ & 10 & 13,7 & 10 & 13,7 & --- & --- \\
\hline $\begin{array}{l}\text { El hombre es el responsable de la } \\
\text { protección }\end{array}$ & 26 & 35,6 & 31 & 42,5 & $0,84(1,145)$ & 0,339 \\
\hline $\begin{array}{l}\text { A mi chico/a no le gusta que use } \\
\text { preservativo }\end{array}$ & 14 & 19,2 & 7 & 10,6 & $2,78(1,132)$ & 0,096 \\
\hline $\begin{array}{l}\text { Prefiero mantener relaciones sexuales } \\
\text { sin condón, aunque me arriesgue a } \\
\text { contraer una enfermedad }\end{array}$ & 6 & 8,2 & 1 & 1,4 & * & 0,058 \\
\hline $\begin{array}{l}\text { Me preocupa más el embarazo que } \\
\text { las infecciones de transmisión sexual }\end{array}$ & 15 & 20,5 & 9 & 12,3 & $1,80(1,146)$ & 0,180 \\
\hline $\begin{array}{l}\text { Me abstendría de mantener relaciones } \\
\text { sexuales por miedo al contagio }\end{array}$ & 18 & 24,7 & 21 & 29,6 & $0,44(1,144)$ & 0,507 \\
\hline $\begin{array}{l}\text { Prefiero mantener relaciones sexuales } \\
\text { con penetración, aunque pueda } \\
\text { contagiarme }\end{array}$ & 13 & 17,8 & 6 & 8,3 & $2,86(1,145)$ & 0,091 \\
\hline
\end{tabular}

Fuente: Elaboración propia a partir de los datos recogidos en el estudio.

$\mathrm{a}_{\mathrm{El}} \chi^{2}$ se calcula sobre los datos con información.

*El $50 \%$ de las casillas tiene una frecuencia esperada menor a 5 . Se usó el test exacto de Fisher.

porcentajes en actitudes como "hace sentirse bien", "está de moda", como así también el bajo porcentaje al cual le molestaba su consumo (Cuadro 5).

Con respecto a la cocaína, el 9,6\% del grupo de intervención y el $17,8 \%$ del grupo control $(p=0,114)$ afirmaba que "estaba de moda" y al $41,1 \%$ del grupo de intervención y al $37,7 \%$ del grupo control les molestaba su consumo. Para el resto de las sustancias, el 1,4\% del grupo de intervención y el $8,2 \%$ del grupo control $(p=0,058)$ opinaban que estaban de moda los alucinógenos y solo les molestaba su consumo al
$35,6 \%$ en el grupo intervención y al 38,6\% del grupo control, mientras que les molestaba el consumo de éxtasis al 43,8\% del grupo intervención $y$ al $35,6 \%$ del grupo control.

Las sustancias consideradas mayoritariamente "nada" o "algo" peligrosas fueron los tranquilizantes, el tabaco, el alcohol y el hachís. El resto de las drogas ilegales (cocaína, éxtasis y alucinógenos) fueron consideradas "bastante" o "muy" peligrosas por la mayoría (Cuadro 6).

Destacan como situaciones viales consideradas "nada" o "algo" peligrosas: no llevar casco cuando se va en bicicleta, cruzar la calle 
Cuadro 4. Grado de acuerdo con actitudes frente al tabaco y al alcohol, en función de su pertenencia al grupo intervención o al grupo control. Porcentajes y frecuencias absolutas. Sevilla, 2009.

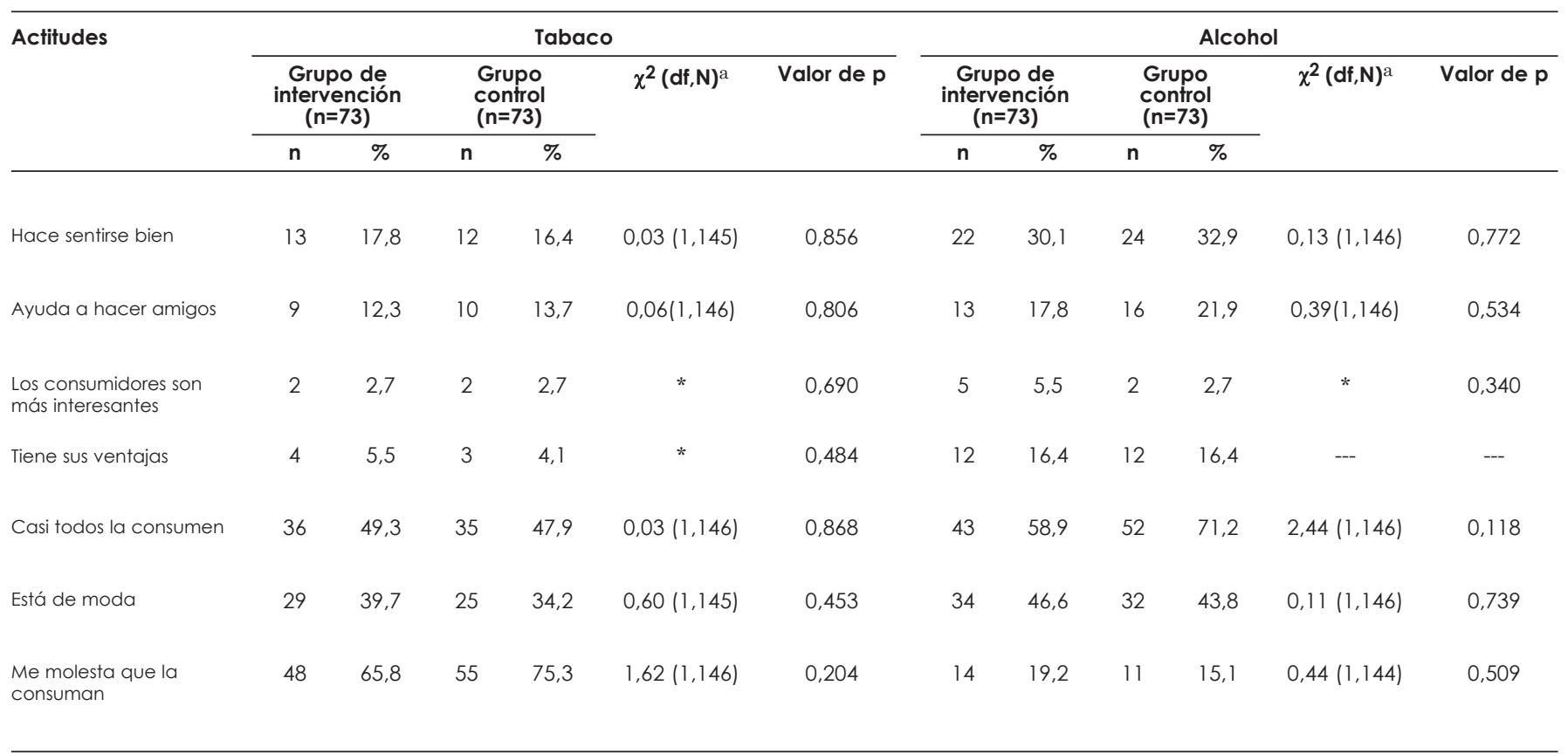

Fuente: Elaboración propia a partir de los datos recogidos en el estudio.

$\mathrm{a}_{\mathrm{El}} \chi^{2}$ se calcula sobre los datos con información.

*El $50 \%$ de las casillas tiene una frecuencia esperada menor a 5 . Se usó el test exacto de Fisher.

Cuadro 5. Grado de acuerdo con actitudes frente a tranquilizantes y hachís de los participantes, en función de su pertenencia al grupo intervención o al grupo control. Porcentajes y frecuencias absolutas. Sevilla, 2009.

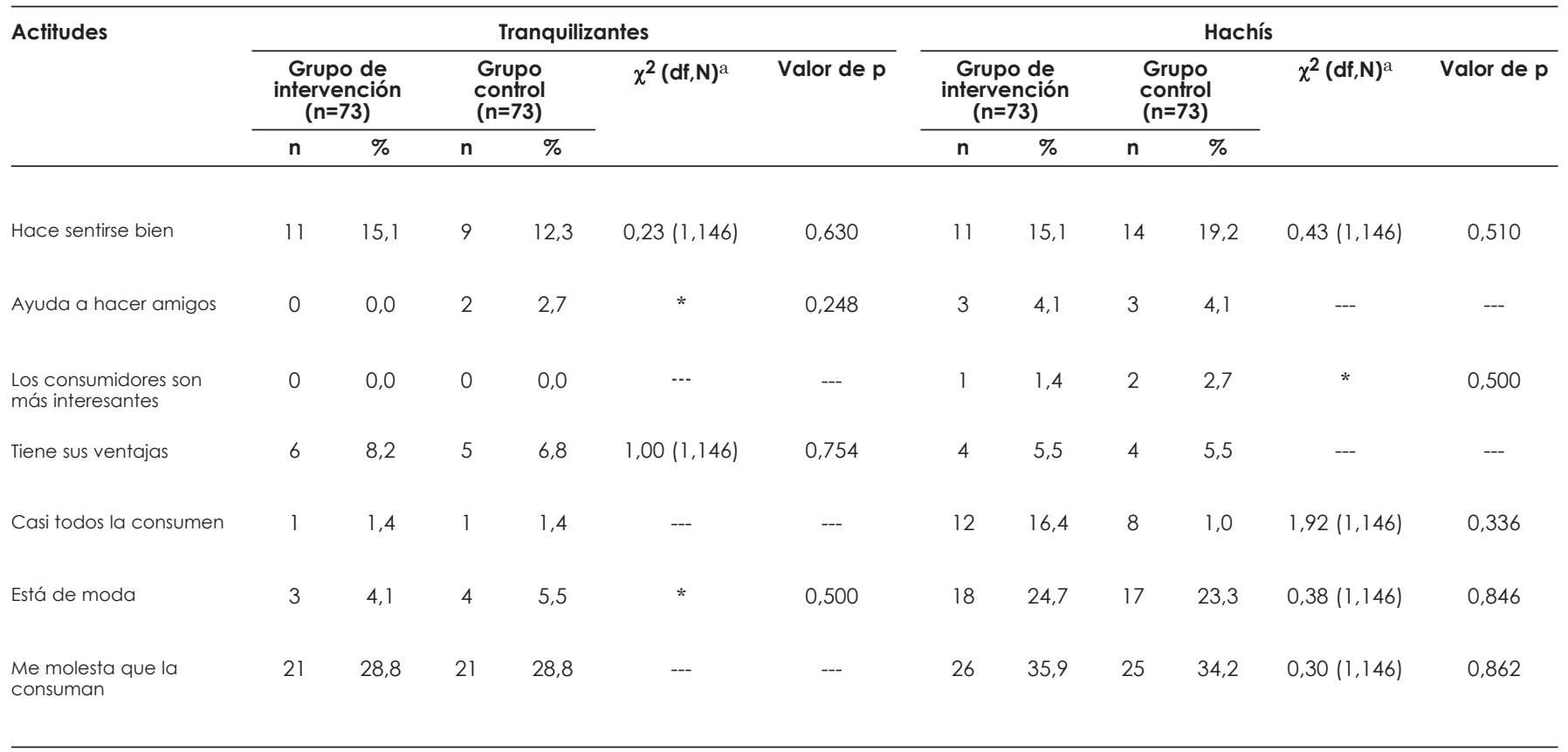

Fuente: Elaboración propia a partir de los datos recogidos en el estudio.

$\mathrm{a}_{\mathrm{El}} \chi^{2}$ se calcula sobre los datos con información.

*El $50 \%$ de las casillas tiene una frecuencia esperada menor a 5 . Se usó el test exacto de Fisher. 
Cuadro 6. Grado de peligrosidad asignado a las sustancias adictivas por los participantes, en función de su pertenencia al grupo intervención o al grupo control. Porcentajes y frecuencias absolutas. Sevilla, 2009.

\begin{tabular}{|c|c|c|c|c|c|c|c|c|c|c|}
\hline \multirow{4}{*}{$\begin{array}{l}\text { Sustancias } \\
\text { adictivas }\end{array}$} & \multicolumn{10}{|c|}{ Grado de peligrosidad } \\
\hline & \multicolumn{4}{|c|}{ Grupo de intervención $(n=73)$} & \multicolumn{4}{|c|}{ Grupo control ( $n=73)$} & \multirow[t]{3}{*}{$\chi^{2}(\mathrm{df}, \mathrm{N})$} & \multirow[t]{3}{*}{ Valor de $p$} \\
\hline & \multicolumn{2}{|c|}{ Nada-algo } & \multicolumn{2}{|c|}{ Bastante-mucho } & \multicolumn{2}{|c|}{ Nada-algo } & \multicolumn{2}{|c|}{ Bastante-mucho } & & \\
\hline & $\mathrm{n}$ & $\%$ & $n$ & $\%$ & $\mathrm{n}$ & $\%$ & $\mathrm{n}$ & $\%$ & & \\
\hline Tabaco & 25 & 34,2 & 48 & 65,8 & 18 & 24,7 & 55 & 75,3 & $1,62(1,146)$ & 0,204 \\
\hline Alcohol & 21 & 28,7 & 52 & 71,3 & 20 & 27,4 & 53 & 72,6 & $0,03(1,146)$ & 0,854 \\
\hline Tranquilizantes & 28 & 38,2 & 45 & 61,8 & 24 & 32,8 & 49 & 67,2 & $0,48(1,146)$ & 0,489 \\
\hline Hachís o marihuana & 11 & 15,1 & 62 & 84,9 & 6 & 8,2 & 67 & 91,8 & $1,66(1,146)$ & 0,197 \\
\hline Cocaína en polvo & 3 & 4,1 & 70 & 95,9 & 1 & 1,4 & 72 & 98,6 & $*$ & 0,310 \\
\hline Éxtasis & 3 & 4,1 & 70 & 95,5 & 1 & 1,4 & 72 & 98,6 & * & 0,310 \\
\hline Alucinógenos & 3 & 4,1 & 70 & 95,9 & 1 & 1,4 & 72 & 98,6 & * & 0,310 \\
\hline
\end{tabular}

Fuente: Elaboración propia a partir de los datos recogidos en el estudio.

*El 50\% de las casillas tiene una frecuencia esperada menor a 5. Se usó el test exacto de Fisher.

por donde está prohibido, circular por una carretera en mal estado, y viajar con un conductor que habla por el móvil (Cuadro 7). Tan solo se identificaron diferencias estadísticamente significativas con respecto al grado de peligrosidad asignado a dos de las trece situaciones viales expuestas: no usar el cinturón de seguridad $(p=0,002$; $r \varphi=0,25)$, e ir en un vehículo cuando el conductor ha consumido alcohol $(p=0,028 ; r \varphi=0,19)$. También se observó una tendencia en el ítem "no llevar casco cuando se va en moto" $(p=0,060)$. EI grado de peligrosidad asignado fue menor en el grupo de intervención.

\section{DISCUSIÓN}

De los resultados se deriva que los participantes, en general, tenían actitudes favorables hacia la adopción de comportamientos saludables con respecto a la alimentación, la sexualidad, las sustancias adictivas o la seguridad vial. Sin embargo, con relación a la alimentación, un importante porcentaje de alumnos se mostraba propicio hacia el consumo diario de dulces o bollería y a saltarse alguna comida al hacer dieta. Estudios previos sugieren una mayor tendencia en el consumo de dulces en la población escolar española $(32,33)$.

Con respecto a la sexualidad, destacaron actitudes poco favorables hacia el uso de preservativo, una alta valoración del coito como expresión de sexualidad, y algunas actitudes sexistas. Esto podría indicar la existencia de un patrón masculino en la actitud hacia la sexualidad de los participantes (34).

La mayoría de las sustancias adictivas fueron consideradas "de moda". El alcohol fue la que mostró un mayor porcentaje de acuerdo en actitudes de aceptación. En un estudio similar realizado en Barcelona (35), también aparece un importante porcentaje de actitudes de aceptación del alcohol. En dicho trabajo, cuando se les preguntó si el alcohol los hace sentir más felices, el 30,6\% responde afirmativamente, el 52,2\% consideraba que el alcohol hace las fiestas más divertidas y el $68,2 \%$ que está bien que se publiciten bebidas alcohólicas. Además estas actitudes se relacionan con lo 
Cuadro 7. Grado de peligrosidad asignado a situaciones relacionadas con la seguridad vial por los participantes, en función de su pertenencia al grupo intervención o al grupo control. Porcentajes y frecuencias absolutas. Sevilla, 2009.

\begin{tabular}{|c|c|c|c|c|c|c|c|c|c|c|}
\hline \multirow[t]{4}{*}{ Situaciones viales } & \multicolumn{10}{|c|}{ Grado de peligrosidad } \\
\hline & \multicolumn{4}{|c|}{ Grupo de intervención (n=73) } & \multicolumn{4}{|c|}{ Grupo control (n=73) } & \multirow[t]{3}{*}{$\chi^{2}(\mathrm{df}, \mathrm{N})^{\mathrm{a}}$} & \multirow[t]{3}{*}{ Valor de $p$} \\
\hline & \multicolumn{2}{|c|}{ Nada-algo } & \multicolumn{2}{|c|}{ Bastante-mucho } & \multicolumn{2}{|c|}{ Nada-algo } & \multicolumn{2}{|c|}{ Bastante-mucho } & & \\
\hline & $\mathrm{n}$ & $\%$ & $\mathbf{n}$ & $\%$ & $\mathbf{n}$ & $\%$ & $\mathrm{n}$ & $\%$ & & \\
\hline $\begin{array}{l}\text { No ponerse el casco cuan- } \\
\text { do va en bicicleta }\end{array}$ & 54 & 74,0 & 19 & 26,0 & 54 & 74,0 & 19 & 26,0 & --- & --- \\
\hline $\begin{array}{l}\text { Cruzar la calle donde está } \\
\text { prohibido }\end{array}$ & 42 & 57,5 & 31 & 42,5 & 35 & 47,9 & 38 & 52,1 & $1,35(1,146)$ & 0,246 \\
\hline $\begin{array}{l}\text { No ponerse el cinturón de } \\
\text { seguridad }\end{array}$ & 9 & $12,3^{*}$ & 64 & 87,7 & 0 & $0,0 *$ & 73 & 100,0 & $* *$ & 0,002 \\
\hline $\begin{array}{l}\text { No ponerse el casco cuan- } \\
\text { do va en moto }\end{array}$ & 4 & 5,5 & 69 & 94,5 & 0 & 0,0 & 73 & 100,0 & $* *$ & 0,060 \\
\hline $\begin{array}{l}\text { Viajar con un conductor que } \\
\text { habla por el móvil }\end{array}$ & 14 & 19,2 & 59 & 80,8 & 10 & 13,7 & 63 & 86,3 & $0,80(1,146)$ & 0,372 \\
\hline $\begin{array}{l}\text { Conducir hablando por el } \\
\text { móvil }\end{array}$ & 13 & 17,8 & 60 & 80,8 & 6 & 8,2 & 67 & 91,8 & $3,00(1,146)$ & 0,085 \\
\hline $\begin{array}{l}\text { Circular a más velocidad de } \\
\text { la permitida }\end{array}$ & 11 & 15,1 & 62 & 84,9 & 7 & 9,6 & 66 & 90,4 & $1,01(1,146)$ & 0,314 \\
\hline $\begin{array}{l}\text { Ir en un vehículo cuando el } \\
\text { conductor consumió alcohol }\end{array}$ & 5 & $6,9 * * *$ & 68 & 93,1 & 0 & $0,0 * * * *$ & 73 & 100,0 & $* *$ & 0,028 \\
\hline $\begin{array}{l}\text { Ir en vehículo cuando el } \\
\text { conductor ha fumado hachís }\end{array}$ & 5 & 6,8 & 68 & 93,2 & 6 & 8,2 & 67 & 91,8 & $0,10(1,146)$ & 0,754 \\
\hline $\begin{array}{l}\text { Ir en vehículo cuando el } \\
\text { conductor consumió drogas }\end{array}$ & 4 & 5,5 & 69 & 94,5 & 1 & 1,4 & 72 & 98,6 & $* *$ & 0,183 \\
\hline $\begin{array}{l}\text { Conducir un vehículo tras } \\
\text { consumir alcohol }\end{array}$ & 3 & 4,1 & 70 & 95,9 & 1 & 1,4 & 72 & 98,6 & $* *$ & 0,310 \\
\hline $\begin{array}{l}\text { Subirse a un vehículo que } \\
\text { está en mal estado }\end{array}$ & 7 & 9,6 & 65 & 89,0 & 9 & 12,3 & 64 & 87,7 & $025(1,145)$ & 0,616 \\
\hline $\begin{array}{l}\text { Circular por una carretera en } \\
\text { mal estado }\end{array}$ & 18 & 27,4 & 54 & 74,0 & 15 & 20,5 & 58 & 79,5 & $0,41(1,145)$ & 0,523 \\
\hline
\end{tabular}

Fuente: Elaboración propia a partir de los datos recogidos en el estudio.

$\mathrm{a}_{\mathrm{El}} \chi^{2}$ se calcula sobre los datos con información.

$* p<0,01$

**El $50 \%$ de las casillas tiene una frecuencia esperada menor a 5 . Se usó el test exacto de Fisher.

$* * * p<0,05$

encontrado en estudios previos, en los que se concluye que el alcohol es la sustancia más consumida entre adolescentes españoles y andaluces, a distancia de otras drogas. Así, en el año 2008 , el $81,2 \%$ de los/as estudiantes de enseñanza secundaria de entre 14 y 18 años había consumido alguna vez en su vida bebidas alcohólicas (29). En Andalucía, en el año 2006, el $57,6 \%$ de estudiantes entre 11 y 17 años también las había consumido (8).

Por otra parte, a pesar de que a la mayoría le molestaba que los demás fumaran tabaco, fueron poco esperanzadores los resultados de este ítem en sustancias como el alcohol y otras drogas, lo cual puede reflejar una alta aceptación del consumo de dichas sustancias entre los participantes.

En el estudio se encontró que el hachís y las drogas legales eran consideradas las menos peligrosas. Por el contrario, las drogas ilegales fueron consideradas peligrosas por la mayoría. Este resultado es similar a lo encontrado por Nebot et al. (35), salvo en el caso del hachís que en dicho estudio fue mayoritariamente considerado mucho o moderadamente peligroso $(92,1 \%)$. El grado de 
peligrosidad asignado a las sustancias adictivas o percepción del riesgo de su consumo está indicado como uno de los factores de riesgo para el uso de las mismas $(29,30)$, lo que queda reflejado en el hecho de que las drogas legales y el hachís sean las más consumidas entre los jóvenes andaluces y españoles. Esta situación podría ser similar con las situaciones viales que han sido consideradas menos peligrosas por los participantes del estudio, tales como no usar el casco cuando se va en bicicleta o cruzar por donde está prohibido.

En las pruebas de homogeneidad no se encontraron diferencias significativas en las características sociodemográficas y en la dinámica familiar. Se observaron tendencias que diferenciaban algunas de las actitudes estudiadas, a veces en dirección al grupo de intervención y otras veces en dirección al grupo control, pero solo se observaron diferencias estadísticamente significativas en la percepción del riesgo de dos de las trece situaciones riesgosas de seguridad vial expuestas, que no llegaron a alcanzar un tamaño del efecto medio. Estas diferencias tal vez pudieran estar relacionadas con la existencia de otras variables perturbadoras no consideradas en este estudio. Generalmente, en los centros educativos, aunque no se lleve a cabo el programa "Forma Joven", suelen realizarse otro tipo de intervenciones. En el caso de la prevención de accidentes de tráfico, es frecuente que acudan a los centros agentes sociales como, por ejemplo, la Asociación para el Estudio de la Lesión Medular Espinal (AESLEME) u otras instituciones como la Guardia Civil de Tráfico. En la investigación se contó con grupos poblacionales "naturalmente formados" y en algunos de los centros pertenecientes al grupo control pueden haberse abordado estos temas, incidiendo en la mejora de la percepción del riesgo del alumnado. En futuros estudios sería interesante profundizar en el grado de implementación de intervenciones de promoción de salud en los centros para mejorar la comprensión de este resultado.

Con frecuencia, los programas de promoción de la salud y prevención de conductas de riesgo no influyen significativamente sobre las actitudes $(19,35,36)$. A pesar de que la familia y los grupos de pares están indicados como los principales agentes en la socialización de estilos de vida saludables (37) y que entre los principios y acciones del programa "Forma Joven" se encuentra el trabajo con estos grupos, durante las entrevistas de planificación realizadas en nuestro estudio se observó que, en los centros participantes del programa no se contó con la participación de madres y padres ni con la figura de mediadores.

Fernández et al. (38), realizaron una revisión sistemática para identificar las principales características de los programas preventivos efectivos durante la adolescencia, destacando los elementos y contenidos a abordar, la metodología a emplear (principalmente activa), la presencia de iguales como mediadores y de las familias, el número y tipo de sesiones, y una evaluación rigurosa, pre y post intervención, para reflejar los efectos positivos. Sin embargo, en los centros estudiados que desarrollan el programa "Forma Joven" no se han encontrado estos elementos de forma sistematizada.

Esto abre el debate acerca de la influencia de estas situaciones sobre los resultados del estudio y el impacto del programa. Se podría afirmar que existe una ruptura entre los principios y las acciones propuestas por el programa "Forma Joven" y la realidad observada en los centros escolares estudiados en cuanto a las actividades Ilevadas a cabo y los agentes sociales implicados, lo que podría estar relacionado con una falta de sistematización en el proceso de implementación.

El estudio se realizó post exposición, ya que el programa llevaba unos años instaurado, por lo que no se pudo obtener información previa sobre las actitudes del alumnado. Es por ello que se incorporó un grupo control en el estudio, y se controlaron estadísticamente algunas variables que pudieran estar influyendo en las actitudes (17-19). Además, se igualaron los grupos de intervención y control con base en la demarcación geográfica, el nivel educativo y el tipo de centro escolar en el que estaban matriculados. En sucesivos estudios sería recomendable ampliar la muestra, complementándola con metodología cualitativa para indagar sobre los discursos de los implicados en el programa (profesionales, familias y los propios adolescentes) con vistas a conocer las dificultades que supone su aplicación, las propuestas de mejora, así como las oportunidades de la promoción de la salud con adolescentes (41), para proponer 
intervenciones que puedan promover un desarroIlo positivo de estos, ayudando a superar las debilidades del programa y aumentando su efectividad.

En el estudio se ha cumplido con los principios bioéticos de beneficiencia, de no maleficiencia, de autonomía y de justicia. Este último puede infringirse en los ensayos comunitarios, cuando se aplica un programa o intervención de forma discriminada a un subgrupo de población, a pesar de sus efectos potenciales sobre su totalidad $(17,40)$. Los centros escolares participantes, previamente habían decidido en forma voluntaria su adscripción o no al programa "Forma Joven" sin que se interfiriera en su implementación.

\section{REFERENCIAS BIBLIOGRÁFICAS}

1. Dirección General de Salud Pública y Participación. Guía Forma Joven. Sevilla: Consejería de Salud de la Junta de Andalucía; 2004.

2. Olavarría L, Borrajo J, Herrera C, Muriel R, González, MJ, Gordillo P, et al. Plan Integral de Atención a la Accidentabilidad 2007-2012 [Internet]. Sevilla: Junta de Andalucía, Consejería de Salud; 2007 [citado 14 jun 2011]. Disponible en: www.juntadeandalucia.es/salud/sites/csalud/galerias/documentos/c_1_c_6_planes_estrategias/plan_accidentabilidad/plan_accidentabilidad.pdf

3. Medina-Mora ME, Natera G, Borges G. Del siglo XX al tercer milenio. Las adicciones y la salud pública: Drogas, alcohol y sociedad. Salud Mental. 2001;24(4):3-19.

4. Schulden JD, Thomas YF, Compton WM. Substance abuse in the United States: findings from recent epidemiologic studies. Current Psychiatry Reports. 2009;11(5):353-359.

5. Saban A, Flisher AJ. The association between psychopathology and substance use in young people: a review of the literature. Journal of Psychoactive Drug. 2010;42(1):37-47.

6. Moreno Rodríguez C, Muñoz Tinoco V, Pérez Moreno PJ, Sánchez Queija I, Granado Alcón MC, Ramos Valverde $\mathrm{P}$, et al. Desarrollo adolescente y salud. Resultados del estudio HBSC-2006 con chicos y chicas españoles de 11 a 17 años. A World Health Organization crossnational study [Internet]. Madrid: Ministerio de Sanidad y Consumo; 2006 [citado 14 jun 2011]. Disponible en: http://www.msps.es/profesionales/saludPublica/pre vPromocion/promocion/saludjovenes/estudioHBS C/nacional_hbsc.htm

7. Escuela Andaluza de Salud Pública. Forma Joven: Una estrategia de salud para adolescentes y jóvenes de Andalucía [Internet]. Granada:
Escuela Andaluza de Salud Pública; 2008 [citado 14 de junio de 2011]. Disponible en: http://mago.easp.es/formajoven/download/RESUMENWEB2.pdf

8. Consejería de Salud. Interrupción voluntaria de embarazo en Andalucía 1999-2008 [Internet]. Sevilla: Junta de Andalucía [citado 14 jun 2011]. Disponible en: www.juntadeandalucia.es/salud/ sites/csalud/contenidos/Informacion_General/p_7 informacion estadistica sanitaria/estadisticas ive? perfil = org\& desplegar $=$ /temas es/P 7 INFORMACION Y ESTADISTICAS SĀNITA $\bar{R} I A S / \& i d i o-$ $\mathrm{ma}=\mathrm{es} \&$ contenido $=/ \mathrm{sites} / \mathrm{csalud} /$ contenidos/Infor macion General/p 7 informacion estadistica sani taria/estadisticas ive)

9. World Health Organization. Diet, nutrition and the prevention of chronic diseases [Internet]. Geneva: WHO; 2003 [citado 14 jun 2011] (WHO Technical Report Series No. 916). Disponible en: http://whqlibdoc.who.int/trs/who trs 916.pdf

10. Consejería de Salud. Plan para la promoción de la actividad física y la alimentación equilibrada [Internet]. Sevilla: Junta de Andalucía, Consejería de Salud [citado 14 jun 2011]. Disponible en: http://www.juntadeandalucia.es/salud/sites/csalud/galerias/documentos/c_1_c 6_planes estrategias/plan alimentacion equilib̄rada/Plan actividad_fisica.pdf

11. Oliva A, Hernando A, Parra A, Pertegal MA, Ríos M, Antolín A. La promoción del desarrollo adolescente: recursos y estrategias de intervención. Sevilla: Junta de Andalucía, Consejería de Salud; 2008.

12. Pertegal MA, Oliva A, Hernando A. Los programas escolares como promotores del desarrollo positivo adolescente. Cultura y Educación. 2010;22(1):53-66.

13. Carvalho SR. Promoción de la salud, "empowerment" y educación: una reflexión crítica como contribución a la reforma sanitaria. Salud Colectiva. 2008;4(3):335-347. 
14. Evolución de la participación en Forma Joven 2001-2010 [Internet]. Sevilla: Junta de Andalucía Forma Joven [citado 14 jun 2011]. Disponible en: http://www.formajoven.org/AdminFJ/doc_cifras_ datos/201151714826231.pdf

15. Listado de puntos Forma Joven [Internet]. Sevilla: Junta de Andalucía, Forma Joven [citado 14 de junio de 2011]. Disponible en: http://www.formajoven.org/pfj/PuntosFormalove n. $a s p x$ ?provincia $=41$

16. Fernández- Ballesteros R. Evaluación de programas. Una guía práctica en ámbitos sociales, educativos y de salud. 2a ed. Madrid: Síntesis; 1996.

17. Cea MA. Metodología cuantitativa, estrategias y técnicas de investigación social. 3a ed. Madrid: Síntesis; 2009.

18. Ramos P, Oliva A, Moreno C, Lorence B, Jiménez $A M$, Jiménez $L$, et al. Los programas escolares para la prevención del consumo de sustancias: Análisis de las claves que determinan su eficacia. Sevilla: Consejería para la Igualdad y Bienestar Social, Universidad de Sevilla; 2010.

19. Jiménez-Iglesias A, Moreno C, Oliva A, Ramos P. Una aproximación a la evaluación de la eficacia de un programa de prevención de drogodependencias en Educación Secundaria en Andalucía. Adicciones. 2010;22(3):253-265.

20. Red de centros docentes [Internet]. Sevilla: Junta de Andalucía, Consejería de Educación [citado 14 jun 2011]. Disponible en: http://www.juntadeandalucia.es/educacion/vscri pts/centros/listado_int.asp

21. Álvarez-Cienfuegos A, Egea F. Aspectos psicológicos de la violencia en la adolescencia. Revista de Estudios de Juventud. 2003;62:37-44.

22. Elzo J. Los valores en la adolescencia. Cuadernos de Pedagogía. 2006;359:18-23.

23. Bartolomé Gutierrez R, Rechea Alberola C. Violencia y conducta antisocial. En: Congreso "Ser adolescente hoy": libro de ponencias. Madrid: Fundación de Ayuda contra la Drogadicción, Ministerio de Trabajo y Asuntos Sociales; 2005. p. 213-224.

24. D'Acremont $M$, Van der Linden $M$. Adolescent impulsivity: Findings from a community sample. Journal of Youth and Adolescence. 2005;34:427-435.

25. Anderson NLR, Nyamathi A, McAvoy JA, Conde F, Conerly C. Perceptions about risk for
HIV/AIDS among adolescents in juvenile detention. Western Journal of Nursing Research. 2001;23(4):336-359.

26. Suárez JC, Navarro FJ, Serra A, Armas A, Aranceta J. Nivel de conocimientos, actitudes y hábitos sobre alimentación y nutrición en escolares de las Palmas de Gran Canaria. Revista Española de Nutrición Comunitaria. 2002;8(12):7-18.

27. Villalbí JR, Nebot M, Ballestín M. Las sustancias adictivas: tabaco, alcohol y drogas no institucionalizadas. Medicina Clínica. 1995;104:784-788.

28. López F, Moral J. Validación de una escala de autoeficacia para la prevención del SIDA en adolescentes. Salud Pública de México. 2001;43:421432.

29. Observatorio Español de Drogas. Encuesta Estatal sobre Uso de Drogas en Enseñanzas Secundarias (ESTUDES) [Internet]. Madrid: Ministerio de Sanidad, Política Social e Igualdad; 2009 [citado 14 jun 2011]. Disponible en: http://www.pnsd.msc.es/Categoria2/observa/estu dios/home.htm

30. Becoña E. Los adolescentes y el consumo de drogas. Papeles del Psicólogo. 2000;77:25-32.

31. Bellón JA, Delgado A, Luna JD, Lardelli P. Validez y fiabilidad del cuestionario de función familiar Apgar-familiar. Atención Primaria. 1996;18(6):289-295.

32. Vázquez $C, \operatorname{Cos} A$, Martínez $P$, Jaunsolo $M A$, Román E, Gómez C, et al. Consumo de alimentos y estado nutricional de los escolares de la Comunidad de Madrid (CAENPE): metodología general y consumo global de alimentos. Nutrición Hospitalaria. 1995;10(1):40-48.

33. Casado MR, Casado I, Díaz GJ. La alimentación de los escolares de trece años del municipio de Zaragoza. Revista Española de Salud Pública. 1999;73(4):501-510.

34. Oliva A, Serra L, Vallejo R. Patrones de comportamiento sexual y contraceptivo en la adolescencia. Infancia y Aprendizaje. 1997;77:19-34.

35. Nebot $M$, Garcia-Continente $X$, Pérez $A$, Ariza C, Espelt A, Pasarín M, et al. Informe FRESC 2008. Taules exhaustives de 4t d'ESO. Barcelona: Agència de Salut Pública de Barcelona; 2010.

36. Fernández $S$, Nebot $M$, Jané $M$. Evaluación de la efectividad de los programas escolares de prevención del consumo de tabaco, alcohol y 
cannabis: ¿qué nos dicen los metaanálisis? Revista Española de Salud Pública. 2002;76(3):175-187.

37. Faggiano F, Vigna-Taglianti FD, Versino $E$, Zambon A, Borraccino A, Lemma P. Prevención del consumo de drogas ilegales en las escuelas. (Revisión Cochrane traducida) [Internet]. La Biblioteca Cochrane Plus 2008 [citado 18 jun 2010];(4). Oxford: Update Software Ltd. Disponible en: http://www.update-software.com/ BCP/BCPGetDocument.asp?SessionID = \%208637 38\&DocumentID $=$ CD003020
38. Castillo I, Balaguer I, García-Merita M, Valcárcel P. El papel de la familia y de los pares en el estilo de vida de los adolescentes. Encuentros en Psicología Social. 2004;2(1):20-26.

39. Minayo MCS. Interdisciplinariedad y pensamiento complejo en el área de la salud [Editorial]. Salud Colectiva. 2008;4(1):5-8.

40. Argimon JM. Métodos de investigación clínica y epidemiológica. 3a ed. Barcelona: Elsevier; 2004.

\section{FORMA DE CITAR}

Lima Serrano M, Neves de Jesus S, Lima Rodríguez JS. Actitudes de adolescentes hacia la salud: evaluación de un programa escolar de promoción de la salud en Sevilla, España. Salud Colectiva. 2012;8(1):47-60.

Recibido el 24 de junio de 2011

Versión final presentada el 19 de octubre de 2011

Aprobado el 14 de noviembre de 2011 